\title{
Does treating hearing loss prevent or slow the progress of dementia? Hearing is not all in the ears, but who's listening?
}

\author{
Stephen M. Stahl \\ ISSUE: \\ Since hearing loss is strongly linked to cognitive decline, treating hearing impairment \\ may have the potential to prevent the onset or slow the progress of dementia.
}

\section{Take-Home Points}

- Numerous hypotheses link hearing loss to cognitive decline and dementia.

- Hearing loss is associated with brain atrophy and neurodegeneration, whereas treating hearing impairment is associated with cortical restructuring and cognitive improvement.

- To the extent that hearing loss may cause cognitive decline, treating hearing impairment could hypothetically prevent the onset or slow the progression of cognitive impairment and dementia by promoting neuroplasticity and curbing neurodegeneration.

\section{Introduction}

More than 5 million individuals in the United States currently suffer from Alzheimer's disease. ${ }^{1}$ Numerous clinical trials have attempted to prevent dementia progression by interfering with beta amyloid deposition in the brain, but the results have been disappointing. ${ }^{2,3}$ If no effective treatment is discovered, by the year 2050 the number of Americans with Alzheimer's disease is projected to reach an alarming figure-nearly 14 million individuals. ${ }^{1}$ In the absence of effective drug therapies for dementia, enhancing neuroplasticity by non-invasive means-such as those hypothesized to occur with physical exercise, from practicing computerized cognitive tasks, or by improving hearing loss—-might be promising short-term alternatives to drugs in order to delay the onset or slow the pace of cognitive decline in Alzheimer's disease. As hearing loss now affects approximately two-thirds of Americans aged 70 or older and is projected to double in adults in the US by $2060,{ }^{4}$ apace with the explosion in the projected incidence of Alzheimer's disease, it certainly seems timely to explore the possibility that treating hearing loss might help to stem the tide of Alzheimer's disease.

\section{What is the evidence that hearing loss correlates} with dementia?

Hearing impairment is independently associated with a $30-40 \%$ acceleration in cognitive decline, ${ }^{5,6}$ as well as an increased rate of all-cause dementia. ${ }^{7,8}$ Specifically, mild hearing impairment increases risk of all cause dementia over a 10-year period by twofold; moderate hearing impairment increases risk of dementia threefold, and severe hearing impairment fivefold. ${ }^{7}$ Since hearing impairment in older adults has been known for decades to be strongly and independently associated with dementia, this has given rise to the provocative hypothesis that age-related hearing impairment may causally contribute to dementia risk. ${ }^{5,9-11}$ If this is true, treating hearing impairment would have the potential to prevent or slow cognitive decline in dementia. ${ }^{9-11}$ Unfortunately, surprisingly little research has tested this possibility.

\section{What is the potential mechanism whereby hearing} loss could cause cognitive decline?

Three overarching theories have been proposed to explain how cognitive decline and hearing loss could 


\section{BRAINSTORMS—Clinical Neuroscience Update}

be related (Table 1)..$^{9-13}$ The first is the "common cause hypothesis," which postulates that both hearing loss and cognitive decline/dementia are the results of a common neurodegenerative process in the aging brain, but that one does not cause the other (Figure 1). If this is the case, treating the common neurodegenerative process might help both conditions, but currently there is no available treatment for whatever neurodegenerative process might be causing both Alzheimer's disease $\mathrm{e}^{2,3}$ and agerelated auditory impairment. ${ }^{4-13}$

The second is the "cascade hypothesis," which suggests that auditory deprivation affects cognition directly via impoverished sensory input, as well as indirectly through a decrease in socialization, which is known to occur in those with hearing loss (Figure 2). Social isolation, loneliness, poor verbal communication, and depression in turn cause cognitive decline. ${ }^{13}$ If so, restoring hearing might not allow hearing loss to "cascade" via these factors into cognitive decline. ${ }^{4-13}$

Table 1. Three hypotheses relating hearing impairment with cognitive decline/dementia

- Common causes: Both hearing loss and cognitive decline have the same neurodegenerative cause.

- Cascade: Auditory deprivation cascades into decreased socialization and depression, as well as into impoverished cortical sensory input, which all cascade in turn into cognitive decline/dementia.

- Cognitive load: Hearing loss causes cognitive resources to be diverted from memory function into auditory processing, creating an excessive cognitive load on higher cortical functions, leading to cognitive decline and dementia.

Figure 1.

Common Cause Hypothesis

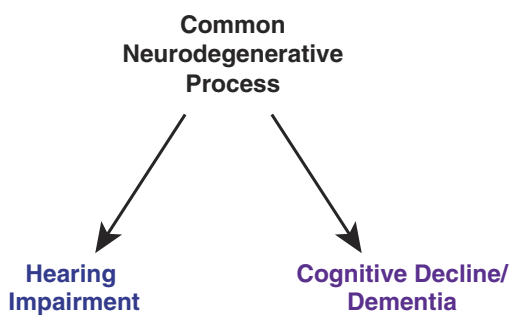

The third hypothesis theorizes that hearing impairment contributes to poorer cognitive functioning by putting an excessive cognitive load on the brain, taxing its structure and leading to more neurodegeneration, or the so-called "cognitive load theory" (Figures 3A and 3B) ${ }^{5-12}$ In this case, hearing loss causes cognitive reserve depletion, leading to cognitive impairment (Figure 3B). For example, recruitment of compensatory brain regions in the prefrontal and temporoparietal cortex during processing of higher level aspects of speech might explain general preservation of language comprehension in the advanced stages of dementia. However, this depletion of cognitive reserve to help compensate for auditory processing deficits might also reduce the neural resources available to other cognitive processes, such as working memory and perceptual processing, thereby increasing the deleterious effects of Alzheimer's disease. ${ }^{5-12}$ The notion here is that the compensatory mental effort required to maintain cognitive function in the setting of hearing loss could divert or monopolize finite and diminishing cognitive resources, resulting in an apparent cognitive decline (Figure 3B). If more cognitive resources are required for auditory perceptual processing of degraded auditory signals, it could be to the detriment of other cognitive processes such as working memory. The cognitive load in a hearing impaired individual would divert cognitive resources away from other cognitive tasks and hypothetically increase cognitive decline and the risk of overt dementia by adding to any brain pathology already resulting from, for example, amyloid/tau accumulation.

Thus, hearing impairment is not good for the brain. Neuroimaging studies demonstrate reduced cortical

\section{Figure 2.}




\section{BRAINSTORMS-Clinical Neuroscience Update}

\section{Figure 3.}

\section{Cognitive Load Hypothesis}

A

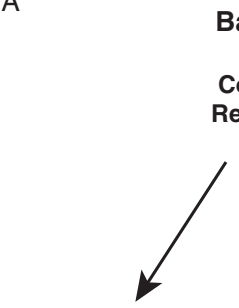

Cognitive Tasks (working memory)
Baseline

Cognitive

Resources

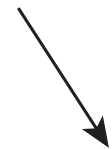

Auditory Processing
B Hearing Loss Increases
the Cognitive Load

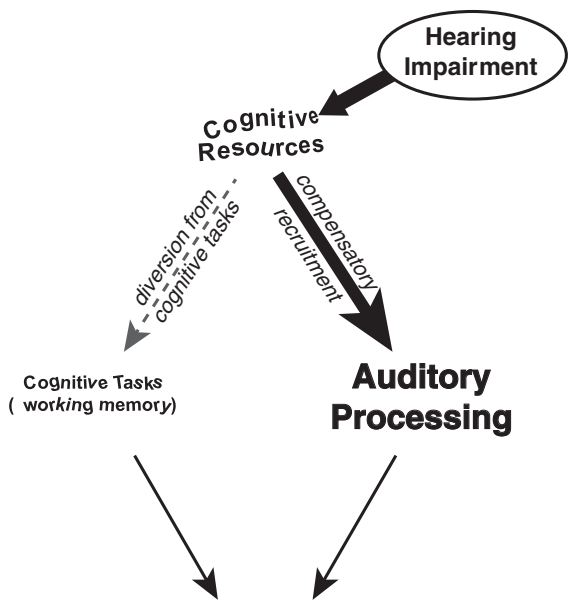

Accelerated Neurodegeneration and Brain Atrophy volume in the auditory cortex as well as accelerated temporal lobe brain atrophy in individuals with hearing loss. ${ }^{9-14}$ We already know from animal studies that impoverished auditory signals and reduced stimulation from an impaired cochlea can cause changes in cortical reorganization and brain morphometry. ${ }^{9-14}$ Human studies also show that hearing impairment causes accelerated rates of brain atrophy, especially in the temporal cortex, ${ }^{9-14}$ a region that is important for semantic memory and sensory integration, as well as spoken language processing, and which is affected in the early stages of both mild cognitive impairment and Alzheimer's disease.

If the relationship between hearing loss and cognitive decline is causal, it follows that rehabilitation of hearing loss might have the potential to impact cognitive decline in elderly patients. The good news is that anatomic studies in fact do show evidence of cortical reorganization in the primary and secondary auditory cortex following hearing restoration after cochlear implantation with the degree of brain plasticity directly associated with the level of speech understanding following implantation. ${ }^{9-14}$ Cochlear implants have also been shown to improve verbal and memory performance. $^{12}$ Early data on the effect of hearing aid amplification on cognition in older adults with or without cognitive impairment is also somewhat promising, but large-scale, randomized, controlled trials are lacking. ${ }^{15-17}$

Why has rehabilitating hearing impairment to prevent or slow cognitive decline not been well studied?

Of all the risk factors for Alzheimer's disease (diabetes, physical activity, age, genetics, environment, lifestyle, etc.), hearing impairment has probably been the least well studied, yet it is the one with potentially a very robust therapeutic approach. Furthermore, hearing aid use is very low, less than $25 \%$ in the US. ${ }^{4}$ Hearing aids are still subject to great stigma and expense, as well as poor coverage by insurance or Medicare. Too often, hearing impairment is an afterthought for clinicians treating aging patients who have risk factors for Alzheimer's disease or who already have signs of cognitive loss. Clinicians should switch from a generally reactive approach (only addressing hearing loss if the patient insists on treatment) to a proactive approach that encourages hearing restoration, which at this point would do no harm and might be helpful for cognition. It is certainly plausible that increased auditory stimulation could reduce the rate of cognitive decline, as well as promote social engagement and reduce cognitive load (Figures 3A and 3B). Uncontrolled studies already suggest a protective effect of hearing aid use on 


\section{BRAINSTORMS —Clinical Neuroscience Update}

cognitive decline ${ }^{15-17}$ but this might be due in part to those who choose to use hearing aids being generally healthier and having higher socio-economic status than those who choose not to use hearing aids. What is needed are large, randomized, controlled trials to determine if treating hearing loss reduces the risk of dementia or slows the rate of cognitive decline.

\section{Summary and Conclusions}

Interventions that could delay the onset of dementia by merely 1 year would result in a more than $10 \%$ decrease in the global prevalence of dementia in $2060 .^{1}$ Treating hearing loss more aggressively is one possibility for delaying the onset of dementia, especially while waiting for effective drugs to be developed. Such trials should be strongly encouraged, and we eagerly await the initiation of such studies and the results.

\section{References:}

1. Alzheimer's Association. 2016. Alzheimer's disease facts and figures. Alzheimer's \& Dementia. 2016; 12(4): 459-509.

2. Godyń J, Jończyk J, Panek D, et al. Therapeutic strategies for Alzheimer's disease in clinical trials. Pharmacol Res. 2016; 68(1): 127-138.

3. Panza F, Seripa D, Solfrizzi V, et al. Emerging drugs to reduce abnormal $\beta$-amyloid protein in Alzheimer's disease patients. Expert Opin Emerg Drugs. 2016; 21(4): 377-391.

4. Gorman AM, Reed NS, Lin FR. Addressing hearing loss in adults in 2060. JAMA Otolaryngol Head Neck Surg. In press. DOI: 10.1001/ jamaoto.2016.4642.
5. Lin FR, Albert M. Hearing loss and dementia-who is listening? Aging Ment Health. 2014; 18(6): 671-673.

6. Lin FR, Yaffe K, Zia J, et al. Hearing loss and cognitive decline among older adults. JAMA Intern Med. 2013; 173(4): 293-299.

7. Lin FR, Metter EJ, O'Brien RJ, Resnick SM, Zonderman AB, Ferrucci L. Hearing loss and incident dementia. Arch Neurol. 2011; 68(2): 214-220.

8. Gallacher J, Ibubaera V, Ben-Shlomo Y, et al. Auditory threshold, phonologic demand and incident dementia. Neurology. 2012; 79(15): 1583-1590.

9. Martini A, Castiglione A, Bovo R, Vallesi A, Gabelli G. Aging, cognitive load, dementia and hearing loss. Audiol Neurootol. 2014; 19(Suppl 1): 2-5.

10. Panza F, Solfrizzi V, Seripa D, et al. Age related hearing impairment and frailty in Alzheimer's disease: interconnected associations and mechanisms. Front Aging Neurosci. 2015; 7: 113.

11. Fortunato S, Forli F, Guglielmi V, et al. A review of new insights on the association between hearing loss and cognitive decline in aging. Acta Otorhinolaryngol Ital. 2016; 36(3): 155-166.

12. Cosetti MK, Pinkston JB, Flores JM, et al. Neurocognitive testing and cochlear implantation: insights into performance in older adults. Clin Interv Aging. 2016; 11: 603-613.

13. Dawes P, Emsley R, Cruickshanks KJ, et al. Hearing loss and cognition: the role of hearing aids, social isolation and depression. PLoS One. 2015; 10(3): e0119616.

14. Lin FR, Ferrucci L, An Y, et al. Association of hearing impairment with brain volume changes in older adults. Neuroimage. 2014; 90: 84-92.

15. Mulrow CD, Aguilar C, Endicott JE, et al. Quality of life changes and hearing impairment: a randomized trial. Ann Intern Med. 1990; 113(3): 188-194.

16. Allen $\mathrm{NH}$, Burns $\mathrm{A}$, Newton $\mathrm{Y}$, et al. The effects of improving hearing in dementia. Age Ageing. 2003; 32(2): 189-193.

17. Kalluri S, Humes LE. Hearing technology and cognition. Am J Audiol. 2012; 21(2): 338-343. 